\title{
Novel method for the production of spin-aligned RI beams in projectile fragmentation reaction with the dispersion matching technique
}

\author{
Y. Ichikawa · H. Ueno · Y. Ishii · T. Furukawa · A. Yoshimi · D. Kameda • \\ H. Watanabe · N. Aoi · K. Asahi • D. L. Balabanski · R. Chevrier • J. M. Daugas • \\ N. Fukuda • G. Georgiev • H. Hayashi • H. Iijima • N. Inabe · T. Inoue · M. Ishihara • \\ T. Kubo • T. Nanao • T. Ohnishi • K. Suzuki • M. Tsuchiya • H. Takeda • M. M. Rajabali
}

(C) Springer Science+Business Media Dordrecht 2012

\begin{abstract}
A novel method to produce spin-aligned rare-isotope (RI) beam has been developed, that is the two-step projectile fragmentation method with a technique of dispersion matching. The present method was verified in an experiment at the RIKEN RIBF, where an RI beam of ${ }^{32} \mathrm{Al}$ with spin alignment of $8(1) \%$ was successfully produced from a primary beam of ${ }^{48} \mathrm{Ca}$, with ${ }^{33} \mathrm{Al}$ as an intermediate nucleus. Figure of merit of the present method was found to be improved by a factor larger than 50 compared with a conventional method employing single-step projectile fragmentation.
\end{abstract}

Y.I. is grateful to the Special Postdoctoral Researchers Program, RIKEN. This work was partly supported by the JSPS KAKENHI (22340071 and 20532089), by the JSPS and MAEE under the Japan-France Integrated Action Program (SAKURA), and by the Bulgarian National Fund (grant DID-02/16).

Y. Ichikawa $(\bowtie) \cdot$ H. Ueno $\cdot$ D. Kameda $\cdot$ H. Watanabe $\cdot$ N. Aoi $\cdot$ N. Fukuda $\cdot$ N. Inabe M. Ishihara $\cdot$ T. Kubo $\cdot$ T. Ohnishi $\cdot$ T. Takeda

RIKEN Nishina Center, 2-1 Hirosawa, Wako, Saitama 351-0198, Japan

e-mail: yuichikawa@phys.titech.ac.jp

Present Address:

Y. Ichikawa

Department of Physics, Tokyo Institute of Technology,

2-12-1 Oh-okayama, Meguro, Tokyo 152-8551, Japan

Y. Ishii · K. Asahi $\cdot$ H. Hayashi $\cdot$ H. Iijima $\cdot$ T. Inoue $\cdot$ T. Nanao $\cdot$ K. Suzuki · M. Tsuchiya Department of Physics, Tokyo Institute of Technology, 2-12-1 Oh-okayama, Meguro, Tokyo 152-8551, Japan

T. Furukawa

Department of Physics, Tokyo Metropolitan University, 1-1 Minami-Ohsawa, Hachioji, Tokyo 192-0397, Japan

A. Yoshimi

Research Core for Extreme Quantum World, Okayama University, 3-1-1 Tsushimanaka, Kita, Okayama 700-8530, Japan 
Keywords Nuclear spin • Nuclear moment • RI beam

\section{Introduction}

The technique of spin orientation in rare-isotope (RI) beam has been played important roles in the study on nuclear structure through the measurement of nuclear electromagnetic moments as well as in application to material sciences $[1,2]$. The mechanism of the spin orientation in a projectile fragmentation (PF) reaction was first revealed in 1990 [3]. The fragmentation of a projectile nucleus in highenergy nucleus-nucleus collisions is described remarkably well by a simple model of "participant-spectator" [4]. In the model, the projectile fragment acquires an angular momentum whose orientation is determined as a function of the momentum of the outgoing fragment. This implies a unique relation between the spin orientation and the direction of the removed momentum, which can be utilized as an obvious means for producing spin-oriented RI beams. One advantage of this method is that the resulting spin orientation does not depend on the chemical or atomic properties of the RI. However, the method also shows a drawback in the sense that the spin orientation thus produced in the PF reaction tends to be partially or completely attenuated in cases that RIs of interest must be produced through the removal of a large number of nucleons from the projectile. Consequently significant magnitude of spin orientation has been produced only for vicinities of a limited set of nuclear species for which high-intensity primary beam are available. To solve this problem, a breakthrough technique, with which highly spin-aligned RI beams can be produced in a promising scheme independently of the mass difference between a projectile and a fragment, has been proposed, that is the two-step PF method with the technique of dispersion matching [5].

\section{Method}

In the proposed method, a nucleus of interest is produced in the second reaction by the one-nucleon removal from a secondary-beam particle so that the spin alignment is expected to be high due to the simplicity of the reaction [3]. An idea of the dispersion matching technique $[6,7]$ in ion optics is combined to the above twostep fragmentation. In a simple scheme of the two-step fragmentation, an RI beam

D. L. Balabanski

Institute for Nuclear Research and Nuclear Energy, Bulgarian Academy of Sciences, 1784, Sofia, Bulgaria

R. Chevrier · J. M. Daugas

CEA, DAM, DIF, 91297 Arpajon, France

G. Georgiev

CSNSM, IN2P3-CNRS, Université Paris-sud, 91405 Orsay, France

M. M. Rajabali

Instituut voor Kern- en Stralingsfysica, K. U. Leuven, Celestijnenlaan 200D,

3001 Leuven, Belgium 


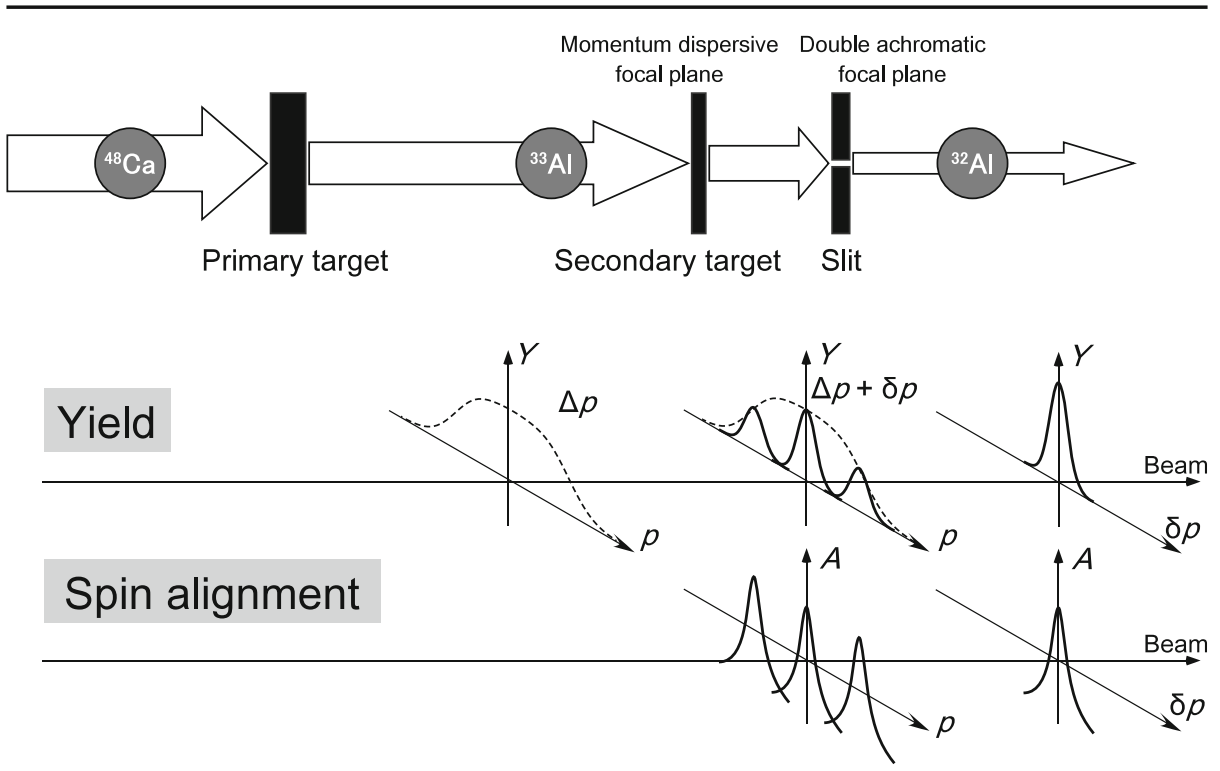

Fig. 1 Scheme of the two-step PF method with momentum-dispersion matching in case to produce ${ }^{32} \mathrm{Al}$ from ${ }^{48} \mathrm{Ca}$ via ${ }^{33} \mathrm{Al}$. The secondary target and a momentum slit are placed at the momentumdispersion focal plane and the double achromatic focal plane, respectively. The graphs below the scheme represent the typical momentum distribution and the corresponding alignment, with abscissas representing the momentum $p$ of ${ }^{32} \mathrm{Al}$. Fulfilling the dispersion-matching condition, the reaction products that acquire equal amounts of momentum change upon the second fragmentation are focused onto a single physical location. The momentum slit can select the momentum change $\delta p$ originating from the second PF reaction independently of the momentum spread $\Delta p$ of the incident beam

of interest is produced after two-fold selections by the momentum slits, the first of which is used to select the momentum of the secondary beam and the second slit determines the outgoing momentum of the secondary beam. The two-fold selections induce the tremendous and unnecessary drop in yield. Here remind that the quantity that determines the spin alignment is solely the momentum change $\delta p$ in the process of fragmentation that produces the nuclei of interest, and the spin alignment is not sensitive to the momentum of the incident nucleus. By placing a secondary target in the momentum-dispersive focal plane and a slit in the double-achromatic focal plane, a single direct selection of the momentum change $\delta p$ can be realized, as shown in Fig. 1. The important point of this technique is that the reaction products that acquire equal amounts of momentum change upon the second fragmentation are focused onto a single physical location. The application of this technique to PF-induced spin alignment can prevent the cancellation of opposite signs of spin alignment caused by momentum spread.

\section{Experiment}

The validity of the method was demonstrated in an experiment performed at RIBF [8, 9], RIKEN. In the reaction at the primary target position F0, ${ }^{33} \mathrm{Al}$ was produced by a PF reaction of a $345-\mathrm{MeV} /$ nucleon ${ }^{48} \mathrm{Ca}$ beam on a ${ }^{9} \mathrm{Be}$ target with 
a thickness of $1.85 \mathrm{~g} / \mathrm{cm}^{2}$, chosen to provide a maximum production yield for the secondary ${ }^{33} \mathrm{Al}$ beam. A wedge-shaped aluminium degrader with a mean thickness of $4.05 \mathrm{~g} / \mathrm{cm}^{2}$ was placed at the first momentum-dispersive focal plane $\mathrm{F} 1$, where the momentum acceptance at $\mathrm{F} 1$ was $\pm 3 \%$. The secondary ${ }^{33} \mathrm{Al}$ beam was introduced to a second wedge-shaped aluminium target with a mean thickness of $2.70 \mathrm{~g} / \mathrm{cm}^{2}$, placed at the second momentum-dispersive focal plane F5. The ${ }^{32} \mathrm{Al}$ nuclei including those in isomeric state ${ }^{32 m} \mathrm{Al}$ were produced through a $\mathrm{PF}$ reaction involving the removal of one neutron from ${ }^{33} \mathrm{Al}$. The ${ }^{32} \mathrm{Al}$ beam was subsequently transported to the focal plane F7 whereby the momentum dispersion between F5 to F7 was tuned to be with the same magnitude and opposite sign as that from F0 to F5, so as to cancel out the momentum dispersion originating from the first PF reaction. The slit at F7 was used to select a region of momentum change at the second $\mathrm{PF}$ as $\delta p / p= \pm 0.15 \%$ about the center of relative momentum distribution.

The ${ }^{32} \mathrm{Al}$ beam was then introduced to an experimental apparatus for timedifferential perturbed angular distribution (TDPAD) measurements, which was placed in a focal plane after the achromatic focal plane F7. The spin alignment is reflected in the amplitude of the changes in anisotropy of the de-excitation $\gamma$ rays emitted from spin-aligned ${ }^{32 m} \mathrm{Al}$ in synchronization with the spin precession in the presence of an external magnetic field. As a result of the experiment, the degree of the spin alignment in the RI beam of ${ }^{32 m} \mathrm{Al}$ was determined to be $8(1) \%$.

A supplementary experiment was also carried out in order to compare the performance of the present method with that of the conventional single-step method, where ${ }^{32} \mathrm{Al}$ was directly produced in a $\mathrm{PF}$ reaction of a ${ }^{48} \mathrm{Ca}$ beam on a $4-\mathrm{mm}$ thick $\mathrm{Be}$ target at the F0 focal plane. In this measurement, the spin alignment was measured to be less than $0.8 \%$ in $2 \sigma$ confidence level. The figure of merit (FOM) for the production of such spin-aligned RI beams should be defined to be proportional to the yield and the square of the degree of alignment. Yields of ${ }^{32 m} \mathrm{Al}$ at the final focal plane were 0.54(5) kcps and 0.87(6) kcps for the two-step PF and the single-step PF measurements, respectively. A primary beam whose intensity was deliberately attenuated by a factor of 1/100 was used in order to avoid saturation in the counting rate at the data acquisition system in the single-step PF measurement. Here, the FOM was compared on the basis of actual effectiveness without correction for the attenuation, in which the resulting FOM for the new method was found to be more than 50 times greater than that of the conventional method employing single-step PF reaction. The superiority in FOM of the new method over the single-step PF method should be even more pronounced for nuclei located farther from the primary beam. In this way the present method is expected to enable us to substantially broaden the accessible scope of spin-aligned RI beams.

Acknowledgements Experiments were performed under Program No. NP0702-RIBF018 at RIBF, operated by RIKEN Nishina Center and CNS, The University of Tokyo. We thank the RIKEN Ring Cyclotron staff for their cooperation during experiments.

\section{References}

1. Frank, M.: On systematics in the ${ }^{19} \mathrm{~F}$ electric hyperfine interactions. Fortschr. Phys. 47, 335-388 (1995)

2. Bharuth-Ram, K.: Hyperfine interaction studies in diamond. Physica B 389, 29-36 (2007) 
3. Asahi, K., et al.: New aspect of intermediate energy heavy ion reactions. Large spin polarization of fragments. Phys. Lett. B 251, 488-492 (1990)

4. Hüfner, J., Nemes, M.C.: Relativistic heavy ions measure the momentum distribution on the nuclear surface. Phys. Rev. C 23, 2538-2547 (1981)

5. Ichikawa, Y., et al.: Production of spin-controlled rare isotope beams. Nature Phys. (2012). doi:10.1038/NPHYS2457

6. Cohen, B.L.: Resolution of accelerator magnetic analyzing systems. Rev. Sci. Instrum. 30, 415-418 (1959)

7. Blosser, H.G., et al.: Ultra-high resolution spectrometer system for charged particle studies of nuclei. Nucl. Instrum. Methods 91, 61-65 (1971)

8. Yano, Y.: The RIKEN RI beam factory project: a status report. Nucl. Instrum. Methods B 261, 1009-1013 (2007)

9. Kubo, T.: In-flight RI beam separator BigRIPS at RIKEN and elsewhere in Japan. Nucl. Instrum. Methods B 204, 97-113 (2003)

10. Robinson, M., et al.: New isomer ${ }^{32} \mathrm{Al}^{m}$. Phys. Rev. C 53, R1465 (1996) 